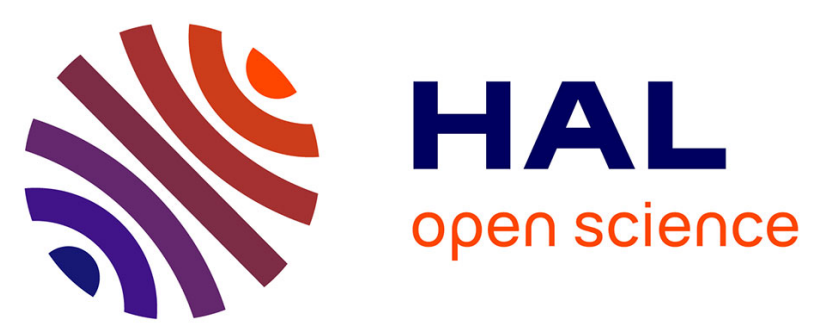

\title{
Magnetoresistance in Fe 0.8 Ga 0.2 thin films with magnetic stripes: The role of the three-dimensional magnetic structure
}

B. Pianciola, S. Flewett, E. de Biasi, C. Hepburn, L. Lounis, M. Vásquez-Mansilla, M. Granada, M. Barturen, M. Eddrief, M. Sacchi, et al.

\section{To cite this version:}

B. Pianciola, S. Flewett, E. de Biasi, C. Hepburn, L. Lounis, et al.. Magnetoresistance in Fe 0.8 Ga 0.2 thin films with magnetic stripes: The role of the three-dimensional magnetic structure. Physical Review B, 2020, 102 (5), 10.1103/PhysRevB.102.054438 . hal-02927053

\section{HAL Id: hal-02927053 https://hal.science/hal-02927053}

Submitted on 15 Sep 2020

HAL is a multi-disciplinary open access archive for the deposit and dissemination of scientific research documents, whether they are published or not. The documents may come from teaching and research institutions in France or abroad, or from public or private research centers.
L'archive ouverte pluridisciplinaire HAL, est destinée au dépôt et à la diffusion de documents scientifiques de niveau recherche, publiés ou non, émanant des établissements d'enseignement et de recherche français ou étrangers, des laboratoires publics ou privés. 


\title{
Magnetoresistance in $\mathrm{Fe}_{0.8} \mathrm{Ga}_{0.2}$ thin films with magnetic stripes: The role of the three-dimensional magnetic structure
}

\author{
B. Pianciola, ${ }^{1}$ S. Flewett, ${ }^{2}$ E. De Biasi,${ }^{1,3}$ C. Hepburn,${ }^{4}$ L. Lounis $\odot,{ }^{4}$ M. Vásquez-Mansilla, ${ }^{1}$ M. Granada, ${ }^{1}$ M. Barturen,${ }^{5}$ \\ M. Eddrief ${ }^{4,6}$ M. Sacchi $\odot,{ }^{4,6,7}$ M. Marangolo, ${ }^{4,6}$ and J. Milano $\circledast^{1,3,6}$ \\ ${ }^{1}$ Instituto de Nanociencia y Nanotecnología, CNEA-CONICET, Centro Atómico Bariloche, (R8402AGP) San Carlos de Bariloche, Argentina \\ ${ }^{2}$ Instituto de Física, Pontificia Universidad Católica de Valparaíso, Avenida Universidad 330, Valparaíso, Chile \\ ${ }^{3}$ Universidad Nacional de Cuyo, Instituto Balseiro, Centro Atómico Bariloche, (R8402AGP) San Carlos de Bariloche, Argentina \\ ${ }^{4}$ Sorbonne Université, CNRS, Institut des NanoSciences de Paris, INSP, UMR7588, F-75005 Paris, France \\ ${ }^{5}$ Instituto de Tecnología, Universidad Argentina de la Empresa, Lima 775, (C1073AAO) Ciudad Autónoma de Buenos Aires, Argentina \\ ${ }^{6}$ LIFAN, Laboratorio Internacional Franco-Argentino en Nanociencias \\ ${ }^{7}$ Synchrotron SOLEIL, L'Orme des Merisiers, Saint-Aubin BP 48, 91192 Gif-sur-Yvette Cedex, France
}

(Received 31 January 2020; revised 27 April 2020; accepted 7 August 2020; published 26 August 2020)

\begin{abstract}
In this work we show the existence of closure domains in $\mathrm{Fe}_{0.8} \mathrm{Ga}_{0.2}$ thin films featuring a striped magnetic pattern and study the effect of the magnetic domain arrangement on the magnetotransport properties. By means of x-ray resonant magnetic scattering, we experimentally demonstrate the presence of such closure domains and also estimate their sizes and relative contribution to surface magnetization. Magnetotransport experiments show that the behavior of the magnetoresistance depends on the measurement geometry as well as on the temperature. When the electric current flows perpendicular to the stripe direction, the resistivity decreases when a magnetic field is applied along the stripe direction (negative magnetoresistance) in all the studied temperature range. Transport calculations in the Ohmic regime indicate that the main source is the anisotropic magnetoresistance. In the case of current flowing parallel to the stripe domains, the magnetoresistance changes sign, being positive at room temperature and negative at $100 \mathrm{~K}$. An intrinsic magnetoresistant contribution arising from the domain walls appears as the most plausible explanation for the observed behavior. We have put in evidence the importance of using x-ray resonant magnetic scattering for the determination of thin-film properties related with the magnetic structure.
\end{abstract}

DOI: 10.1103/PhysRevB.102.054438

\section{INTRODUCTION}

Ferromagnetic thin films characterized by weak stripe domain patterns feature some peculiar properties that open perspectives for their use in magnonic devices. Recently, Liu et al. [1] have shown that in these films it is possible to control the magnetization direction by electrical currents, injecting current densities one order of magnitude lower than what is typically needed when relying on the spin torque effect. They observed also that the attenuation of a magnon current is highly dependent on its direction with respect to the stripe orientation.

Some years ago, the presence of striped magnetic pattern was reported in $\mathrm{Fe}_{1-x} \mathrm{Ga}_{x}$ thin films [2,3] and was ascribed to the presence of a perpendicular-to-the-film magnetic anisotropy (PMA). PMA induces a magnetic easy axis along the surface normal and leads to the appearance of a magnetic structure within the film that is more complex than the usual in-plane configuration imposed by magnetostatics [4]. The $Q$ parameter (defined as $Q=\frac{2 K_{\mathrm{PMA}}}{\mu_{0} M^{2}}$, where $K_{\mathrm{PMA}}$ is the strength of the PMA and $\frac{\mu_{0} M^{2}}{2}$ is the demagnetizing energy $E_{\mathrm{dem}}$, for a magnetic thin film) helps us to measure how far the system is from a fully in-plane magnetic configuration $Q<0$. If $Q>1, K_{\mathrm{PMA}}$ overcomes $E_{\mathrm{dem}}$, so the magnetization points perpendicular to the thin film. However, if $0<Q<1$, an intermediate state exists where PMA competes with the magnetostatic energy. In this case, the film presents stripe-shaped magnetic domains with a complex magnetic structure [5]. This periodically modulated arrangement presents domains with the magnetization vectors pointing along the three spatial directions within the sample. This particular domain structure can be set via a simple magnetic procedure: By applying a saturating magnetic field in the sample plane and then releasing it, stripe domains appear along the direction of the previously applied field. A fraction of the magnetic moments remains along the direction of the most recent saturating field ( $w$ domains), another fraction points perpendicular to the film plane ( $s$ type), and, finally, closure domains $(c)$ appear to reduce the stray magnetic field. The localization of each kind of magnetic domain is sketched in Fig. 1(a). In spite of being predicted analytically [6] and also obtained via micromagnetic calculations [7], a quantitative experimental procedure to study the complete domain structure to nanometer resolution has still not been fully developed. There has been much work done to characterize the domain structure of magnetic thin films in both two and three dimensions as shown in Refs. [8,9] and references contained within, being soft $\mathrm{x}$ rays frequently the probe of choice. 

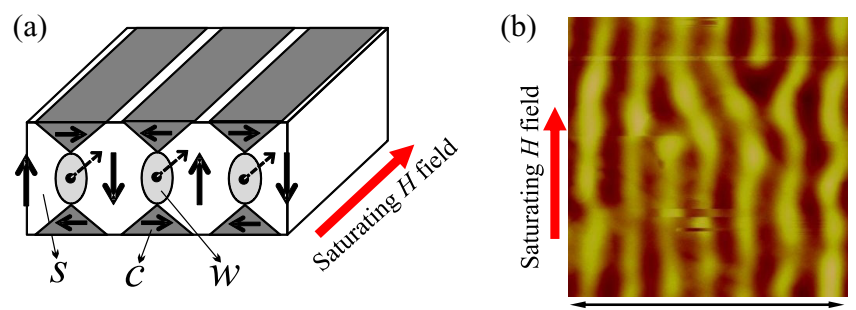

$1 \times 10^{3} \mathrm{~nm}$

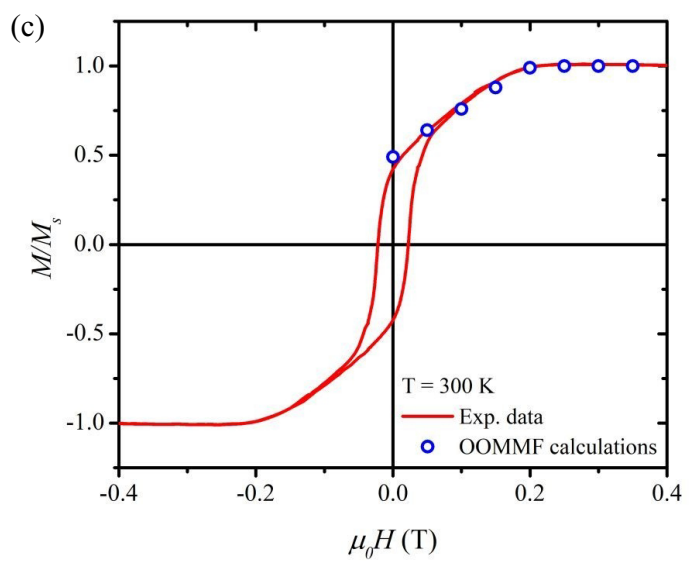

FIG. 1. (a) Schematic view of domains that compose the stripe pattern after the saturating field was applied. (b) MFM image of the stray field generated by the stripe pattern. (c) $M$ vs $H$ loop along the saturating field previously applied, where the characteristic linear behavior of the stripe domains at lower fields is observed in the experimental data (red line) as well as in the OOMMF calculations (blue circles).

The interest in the magnetotransport properties of striped systems started in the the 1990s with the goal of studying giant magnetoresistance (GMR) [10]. However, the GMR ratio is negligible because of the domain walls that separate the magnetic regions of those samples were not sharp enough to provoke a detectable spin-dependent scattering. Nevertheless, it was the starting point for studying the different contributions to the magnetoresistance (MR) that are present in this type of system, such as anisotropic magnetoresistance (AMR), Lorentz magnetoresistance (LMR), and domain wall magnetoresistance (DWMR) [11,12], being this last contribution to MR still a source of debate. DWMR measures how the electronic spin tracks the change of the magnetization across the wall and how it is reflected in the resistance. However, the intrinsic DWMR can be masked for extrinsic factors, such as MR sources present in the magnetic domains that keep on contributing at the DW's, i.e., AMR and LMR, or additional effects related with the stripe geometry and orbital motion of the electrons close to the DW's [12]. Experimentally, the DWMR has not presented the same behavior among the materials where it has been studied, specifically, it can present both positive or negative contributions to the resistivity [13-16]. Theoretically, semiclassical and quantum models were proposed in order to explain its origin using very different approaches, resulting in dissimilar predicted behaviors [16-21].

In Ref. [22], some of the authors have studied the magnetotransport properties of $\mathrm{Fe}_{1-x} \mathrm{Ga}_{x}$, in order to evaluate the behavior of the MR as a function of temperature in different measurement geometries [as depicted in Fig. 4(a)]. We found that at room temperature the sign of the MR depends on the measurement geometry: for the case of electric current flowing perpendicular to stripe direction, the MR (MR-CPW) is negative while, when the current flows along the stripe direction, the MR (MR-CIW) is positive. Moreover, MR-CIW changes its sign at a temperature lower than $300 \mathrm{~K}$.

In this work, we experimentally observe the existence and estimate the size of closure domains in $\mathrm{Fe}_{1-x} \mathrm{Ga}_{x}$ thin films via $\mathrm{x}$-ray resonant magnetic scattering (XRMS). We also study the electrical transport in the Ohmic regime as a function of an applied field when stripes are present. Finally, by considering the magnetic configuration obtained by means of micromagnetic simulations, we correlate the existence of closure domains with the AMR behavior. From the analysis of the experimental data and simulations, we observed that the role played by the DWMR depends on the relative direction of the electric current with respect to stripes.

\section{EXPERIMENTAL DETAILS AND PRELIMINARY CHARACTERIZATION}

Epitaxial $\mathrm{Fe}_{1-x} \mathrm{Ga}_{x}$ samples were grown by molecular beam epitaxy on $c(2 \times 2) \mathrm{Zn}$-terminated $\mathrm{ZnSe}$ epilayers onto GaAs(001) substrates [23,24]. After growth, the films were covered by a protective 3-nm gold capping layer. Details of the growth are given in Ref. [25]. We fabricated 84-nm-thick samples with a Ga concentration of $x=(0.20 \pm 0.02)$. Such concentration was determined by means of $\mathrm{x}$-ray photoelectron spectroscopy (XPS) and confirmed by Rutherford backscattering (RBS) and energy dispersive $\mathrm{X}$-ray spectrometry (EDX). $\mathrm{X}$-ray diffraction (XRD) experiments were performed using $\mathrm{Cu} K_{\alpha}$ radiation in a Philips X'Pert MRD diffractometer [25]. The XRD results show a tetragonal structure which the lattice parameter corresponding to the growth direction presents a elongation of $\sim 2 \%$ with respect to the in-plane lattice parameter. This elongation is the responsible of the existing PMA in our samples [3].

Before performing the experiments, the stripe domains were set, following the procedure mentioned in Sec. I, along a desired sample plane direction. In order to determine the existence and estimate the size of closure domains in the $\mathrm{Fe}_{1-x} \mathrm{Ga}_{x}$ thin films, we carried out XRMS experiments at the Circular Polarization beamline of the ELETTRA synchrotron, using the IRMA scattering chamber [26]. The photon energy was set to $707 \mathrm{eV}\left(\mathrm{Fe}-L_{3}\right.$ resonance, $\left.2 p_{3 / 2} \rightarrow 3 d\right)$ and we used circular polarization of both helicities produced using a helical electromagnetic wiggler source. The scattered intensity was measured using a two-dimensional (2D) detector formed by microchannel plates and a resistive anode.

For the study of the magnetotransport properties, the electrical resistance was measured in a standard four-probe configuration, with collinear contacts along the [110] $\mathrm{Fe}_{0.8} \mathrm{Ga}_{0.2}$ crystalline direction. The voltage contacts were separated by $1.5 \mathrm{~mm}$, so the effective size of the sample was much larger than the stripe period. The measurements were performed with a maximum dc electric current of $10 \mathrm{~mA}$, which gives a current density of $\sim 0.1 \mathrm{GA} / \mathrm{m}^{2}$, much lower than the current densities needed to induce domain wall displacement 
(e.g., some $\mathrm{TA} / \mathrm{m}^{2}$ for $\mathrm{Ni}_{81} \mathrm{Fe}_{19}$ [27]). Thus, we should not expect the electric current to affect the magnetic configuration in any way. The field dependence of the resistivity was measured with the magnetic field applied perpendicular and parallel to the electric current as sketched in Fig. 4(a). In that way, we were able to determine the MR in CPW and CIW configurations.

Additional magnetization measurements were performed in a superconducting quantum interference device (SQUID) and a vibrating sample magnetometer (VSM). In Figs. 1(b) and 1 (c) we show preliminary magnetic characterization of the measured samples. Figure 1(b) shows a magnetic force microscopy (MFM) image. From this picture we can determine a spatial period of the stripes $\lambda_{S} \sim 150 \mathrm{~nm}$. In Fig. 1(c), we display the $M$ vs $H$ loop obtained by VSM at room temperature. We can observe the typical linear behavior at low field, which is a fingerprint of the presence of stripe domains. Also, we performed micromagnetic simulations via the OOMMF package [28] in order to determine the magnetic structure of the studied systems. The saturation field $\left(H_{s}\right)$ and the linear behavior at low field of the hysteresis loop were adjusted [as shown in Fig. 1(c)] using the following parameters: $M_{s}=1.4 \times 10^{5} \mathrm{~J} / \mathrm{m}^{3}, K_{\text {PMA }}=3.5 \times 10^{5} \mathrm{~J} / \mathrm{m}^{3}$, and $A=2.5 \times 10^{-11} \mathrm{~J} / \mathrm{m} . M_{s}$ and $K_{\mathrm{PMA}}$ are in agreement with those experimentally obtained [2,3], while the adjusted $A$ value is larger than previously reported [7]. Using these parameters, we obtained a stripe pattern with a period of $\sim 140 \mathrm{~nm}$. It is important to note that the in-plane anisotropies that this system presents [3] were not taken into account in the micromagnetic calculations. These contributions can be considered negligible (whose anisotropy values are less than $1 \times 10^{4} \mathrm{~J} / \mathrm{m}^{3}$ ) with respect to the magnetostatic anisotropy imposed by the stripe arrangement which is $\sim 2.5 \times 10^{5} \mathrm{~J} / \mathrm{m}^{3}$. In Fig. 1, we show that $H_{s}$ and the remnant magnetization $M_{r}$ obtained via OOMMF (blue circles) present a very good agreement with the experimental results. From the calculated magnetic structure we performed simulations in order to complement the experimental results obtained by XRMS and MR measurements. For the calculations we considered a sample $84 \mathrm{~nm}$ thick and with surface area $570 \times 570 \mathrm{~nm}^{2}$, using periodic boundary conditions to avoid size effects along the sample plane. The sample was discretized into cubic cells of $3.5 \mathrm{~nm}$, which is smaller than the magnetic exchange length given by $\ell_{\mathrm{ex}}=\sqrt{A / K_{\mathrm{PMA}}} \sim 8 \mathrm{~nm}[29]$.

\section{RESULTS AND DISCUSSION}

\section{A. Magnetic structure}

In Fig. 2(a), we sketch the geometry of the XRMS experiment at the $\mathrm{Fe}-L_{3}$ edge with the stripes aligned within the scattering plane. Figures 2(b) and 2(c) show images obtained using a circularly polarized incident beam with either positive $(C+)$ or negative $(C-)$ helicity. At the center of both pictures a large specular reflection peak is present. Marked in red one can observe the first-order diffracted peaks. Note the very high degree of asymmetry observed between the opposite helicities, however, given the partial swamping of the magnetic peaks by the dominant specular peak, the exact degree of this asymmetry is difficult to determine from our data [30]. As previously demonstrated by Dürr et al. [31],
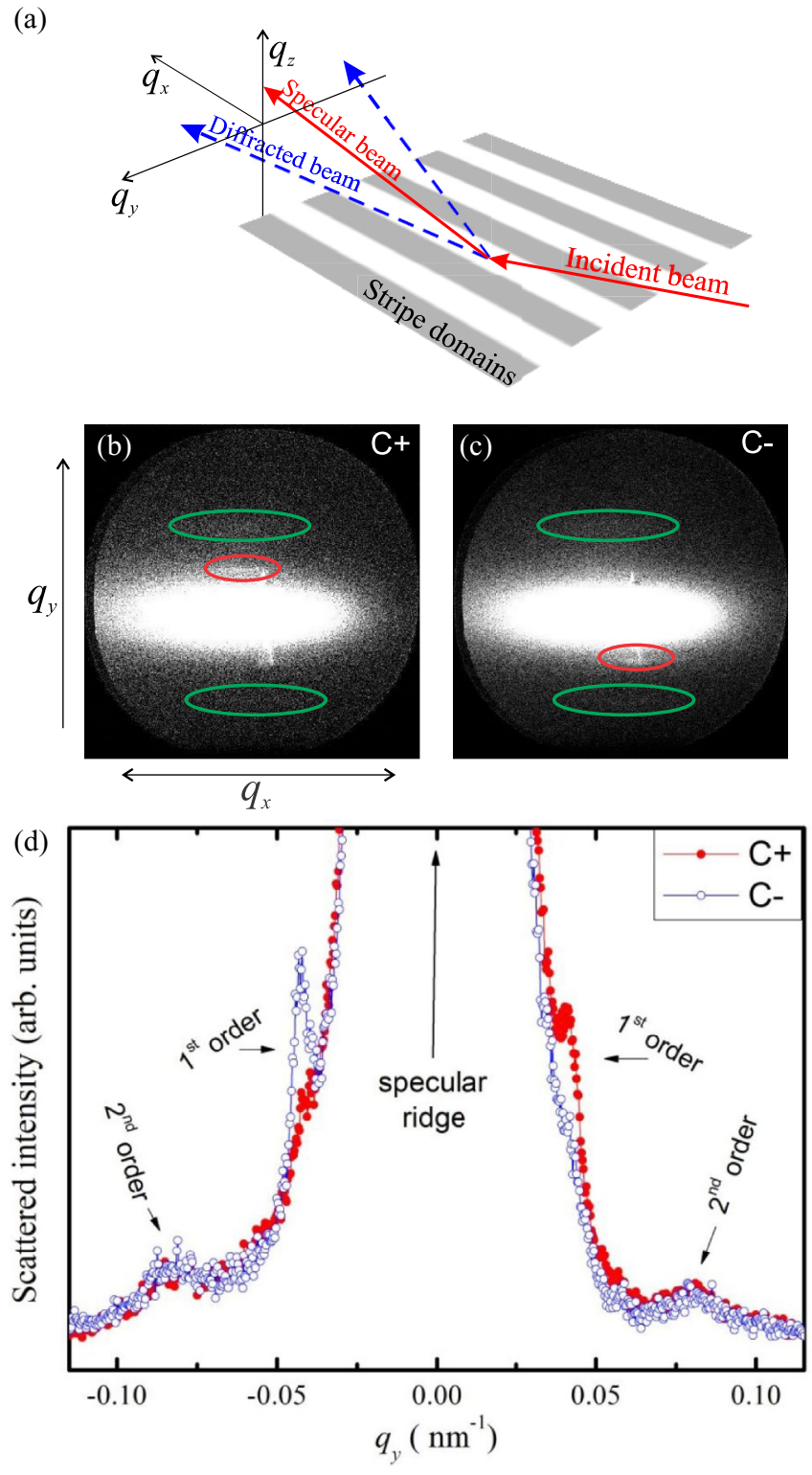

FIG. 2. (a) Measurement geometry. (b) and (c) images of the spots corresponding to the specular, first (marked in red) and second order (marked in green) diffracted peaks for $\mathrm{C}+$ and $\mathrm{C}$ - respectively. At an incidence angle $\omega=16.3^{\circ}$, the $q_{x}$ and $q_{y}$ ranges covered by the detector are approximately $q_{x}= \pm 0.045 \mathrm{~nm}^{-1}$ along the $q_{y}=0$ line and $q_{y}= \pm 0.175 \mathrm{~nm}^{-1}$ along the $q_{x}=0$ line. $(d)$ Peaks transverse section.

this asymmetry is due to the presence of a mixed out of plane/in-plane magnetization at the sample surface (when XRMS is performed in reflection geometry, it probes the magnetic structure mainly of the first monolayers below the surface). In this situation, the periodic structure as seen by the beam consists of a repeating "up, right, down, left" pattern, with the up and down components sensitive to the polar magneto-optical Kerr effect (MOKE) component, and the left and right components sensitive to the transverse MOKE component. As such, the up and down sections produce a scattering signal from the $\sigma$ to $\pi$ and $\pi$ to $\sigma$ polarizations, and the transverse regions of the sample scatter from $\pi$ to $\pi$ 
polarization. Each of these components alone would produce a symmetric diffraction pattern due to an intensity shift and $\pi$ phase shift between the up and down or left and right components of the magnetization, however, under circular polarization where the two linear polarizations are superimposed with a $\pi / 2$ phase shift between, their coherent addition and resulting interference produces the characteristic asymmetric diffraction in the presence of a chiral spatial distribution of the magnetization. Furthermore, we can observe the appearance of second-order peaks (marked in green) whose relative intensity appears not to depend on the beam polarization, although according to analysis they are of magnetic origin and likely due to a partial protrusion of uniformly oriented Bloch walls to the surface layer probed by the $x$ rays. Such uniformly oriented Bloch walls produce a periodic structure in the longitudinal magnetic component with half the period of the principal out of plane and transverse magnetic structure, thus giving rise to the presence of a second-order magnetic peak. In this case, however, the peak is due to the longitudinal MOKE signal, which like the polar MOKE signal produces scattering in the $\sigma$ to $\pi$ and $\pi$ to $\sigma$ directions. Unlike in the case of the first-order scattering peaks, in this case there is no interference between the two linear polarization states which make up the circular polarization state, thus giving rise to a symmetric diffraction signal at second order. Such secondorder peaks are currently subject to more detailed study, with results expected to soon be submitted for publication [32].

Figure 2(d) compares line profiles along $q_{y}$ drawn across the magnetic peaks of the 2D images in Figs. 2(b) and 2(c). A polarization dependence of opposite sign is clearly observed for the two first-order magnetic peaks originating from the striped pattern, this being the classic signature of a chiral magnetic domain morphology. To model the observed scattering behavior, we started from the micromagnetic simulations mentioned before and, using the recipe of Flewett et al. [33-35], generated an artificial 2D disordered stripe domain pattern over a $512 \times 512 \mathrm{~nm}^{2}$ surface at $5 \mathrm{~nm}$ pixel size. We then used the generalized Fresnel formulas, derived in Ref. [36], to calculate the reflection (and transmission) coefficient tensor at each point of the surface of the simulated stripe domain sample, for each incident beam angle, and magnetization direction.

Due to limitations of computational power and to the nonlinear scaling of the micromagnetic simulations, these simulations must be performed over an area much smaller than the incident beam size. Diffraction is, however, determined by the transverse coherence length of the beam, which in our case is about $1 \mu \mathrm{m}$ in size [37-39]. The algorithm of Flewett et al. [33-35] used for extending a reduced size micromagnetic simulation over a larger area assumes that the magnetization vector depends only upon the distance from any given domain wall and on the spatial orientation of such a wall (Bloch, Nèel, or intermediate). This simulation process allows for the simulation of any domain pattern from perfectly ordered stripes to disordered worm domains. In this case we have utilized a partially ordered stripe pattern, which produces broadened peaks with the possibility of doublets in the case of coherent illumination as used in the simulation.

The final step in the simulation process is to calculate the scattering pattern, which is performed simply using Fresnel propagation for a Gaussian beam reflecting from the sample surface according to the already computed array of reflection coefficients. The different polarization steps are treated separately, and finally combined in the calculation of the scattered intensity. In most other XRMS applications, a multilayer sample is used which would require the calculation of reflection coefficients for each and every interface, along with a depthdependent summation considering the attenuation of the beam within the sample. In our case, however, with a single-layer sample, the only appreciable reflection occurs at the upper surface greatly simplifying the calculation.

In Fig. 3(a), we show the simulated magnetic diffraction pattern integrated along the direction parallel to the stripes using the micromagnetic simulation with parameters $M_{s}=$ $1.4 \times 10^{5} \mathrm{~J} / \mathrm{m}^{3}, K_{\mathrm{PMA}}=3.5 \times 10^{5} \mathrm{~J} / \mathrm{m}^{3}$, and $A=2.9 \times$ $10^{-11} \mathrm{~J} / \mathrm{m}$ (solid line). The surface magnetization of this simulation is $30 \%$ out of plane ( $s$-type domains at surface), $15 \%$ inplane parallel to the incident plane ( $w$-type), and 55\% in-plane perpendicular to the incident beam ( $c$-type, closure domains). For comparison, in dashed lines, we show the simulation for a fictitious sample where the parameters were modified with respect to those in agreement with the experimental data. In this case we create a 40-nm-thick sample with the same value of $M_{s}$, and with $K_{\mathrm{PMA}}=7.0 \times 10^{5} \mathrm{~J} / \mathrm{m}^{3}$ and $A=2.9 \times 10^{-11}$ $\mathrm{J} / \mathrm{m}$. Due to the higher $K_{\mathrm{PMA}}$ value an out-of-plane component of 59\% was observed, with $37 \%$ in plane perpendicular to the beam, and $4 \%$ in plane parallel to the beam. This value of the in-plane/out-of-plane ratio is reflected in the fact that the asymmetry of the reflection peaks for the 40-nm sample in Fig. 3(a) is near to its theoretical maximum for this level of stripe disorder. The simulations performed with the experimental parameters exhibit a lower degree of asymmetry in accordance with its greater in-plane surface component compared with that of the fictitious sample. The aim of the XRMS measurements and simulations was to estimate the out-of-plane/in-plane ratio present, to which effect we additionally simulated the asymmetry ratio as a function of outof-plane component using a faster simulation script replacing the micromagnetic simulations with an analytical form of the Nèel-type domain walls given by $M_{z}=\tanh (x / w)$ where $w$ is the domain wall width and $x$ is the perpendicular distance from the domain wall. It is important to mention that the closure domains play the role of the domain walls in this case. $M_{x}$ was chosen in order to maintain a constant magnitude of the magnetization vector $M$. In Fig. 3(b), we show the asymmetry calculated as a function of the domain wall width $w$, using the same degree of disorder as for the experimental case. Here, we observed that the maximum asymmetry ratio is reached for a 20-nm-wide domain wall [40]. In the process of performing these simulations we also observed that the asymmetry ratio depended upon the degree of stripe disorder, with lower levels of disorder exhibiting greater levels of asymmetry. To our knowledge, this relation has not been systematically studied in the literature and could warrant investigation. From these experimental results and corresponding simulations we may conclude that there is unequivocal evidence of a chiral Nèel domain structure present on the surface of the sample, and we estimate that the experimental out-of-plane component to be between the two cases studied with simulations shown in Fig. 3, i.e., with between $30 \%$ and $60 \%$ out of plane. The 

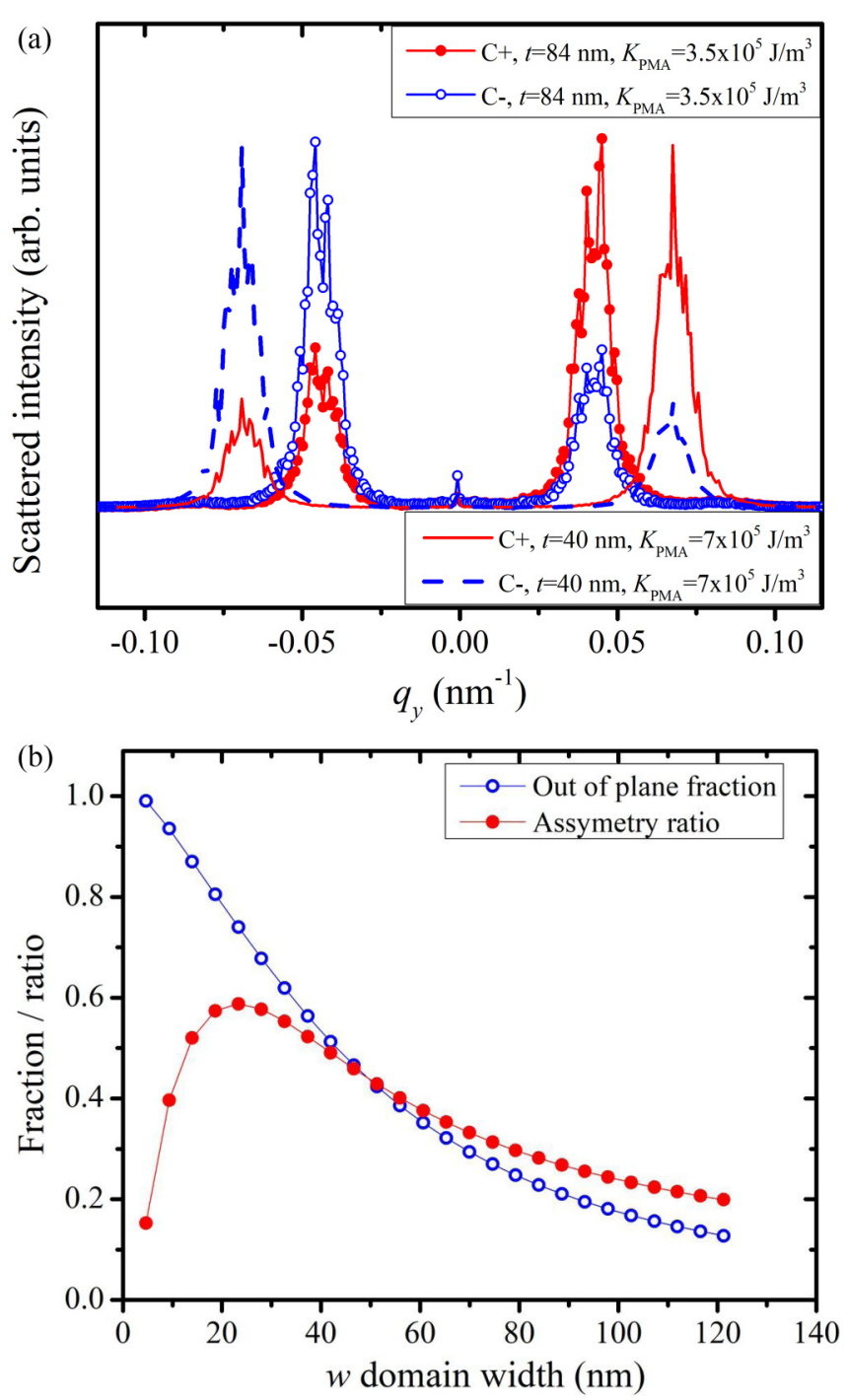

FIG. 3. (a) Scattered intensity vs $q$ calculated from the magnetization structure calculated by OOMMF. The doublet present in the scattering peak for the 84-nm-thickness sample is due to simulated disorder included in the simulation. Had perfect stripes been simulated, the scattering peaks presented would have taken the form of delta functions. (b) Calculated asymmetry ratio and out-of-plane magnetization component as a function of the domain wall width. At either very thin or very thick domain walls, the magnetization distribution is either out of plane or in plane, giving rise to only polar or transverse MOKE components, respectively. In order to produce an asymmetric scattering pattern when illuminated with circularly polarized $\mathrm{x}$ rays, constructive interference is required between the polar and transverse MOKE components, and such interference is greatest at intermediate values of the domain wall width.

very high uncertainty range stated here is due to the high background from the specular reflection peak which made estimating accurately the asymmetry ratio almost impossible. Readers should note that these figures refer only to the surface layer, and that the out-of-plane fraction of the bulk is likely to be higher due to the classic triangular shape of the closure domains in samples of this type.
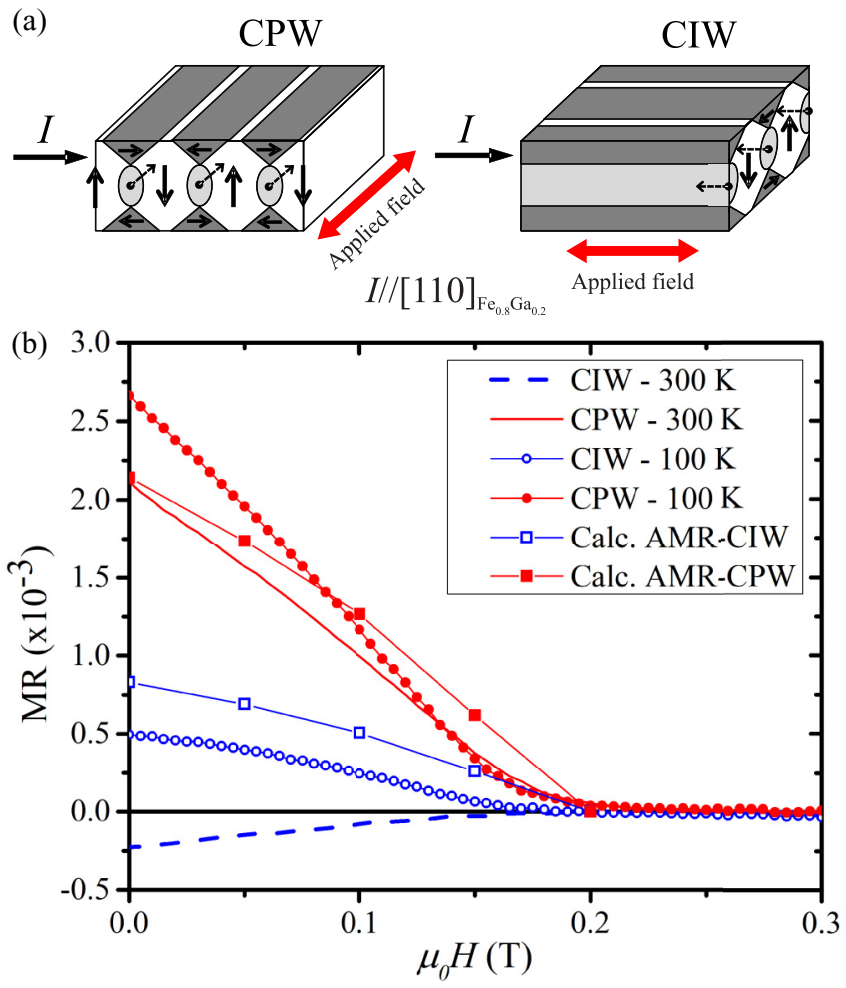

FIG. 4. (a) Geometries used for magnetotransport measurements. (b) MR ratio in both measurement geometries (CPW and CIW) at 300 and $100 \mathrm{~K}$. The lines with squared symbols indicate the AMR calculated from simulations.

The weak reflection signal in this case can be due to the fact that we were working with a single-layer and not a multilayer sample where the reflection signal can be greatly amplified at certain Bragg angles. After the beam time and shortly before submission of this paper, similar samples were characterized using transmission geometry XRMS [33], which would have likely been a better candidate than reflection geometry XRMS for samples of this type due to a better sensitivity to the bulk that is not screened by the surface contribution of the film.

\section{B. Magnetotransport}

In Ref. [22], we have studied the magnetotransport properties of this system as a function of temperature for two geometries as depicted in Fig. 4(a). In the left panel of that figure we sketch the geometry where the electric current flows perpendicular to the stripe direction (CPW geometry), and in the right panel we show the geometry where the current flows parallel to the stripe (CIW one). Figure 4(b) displays the measured $\mathrm{MR}$ ratio (defined as $\mathrm{MR}=\frac{\rho(H)-\rho_{\text {sat }}}{\rho_{\text {sat }}}$ ) for the case of CPW and CIW geometries at $T=300$ and $100 \mathrm{~K}$, previously reported in Ref. [22]. In the case of CPW geometry (red line), we observe that MR-CPW decreases with the applied field irrespective of the temperature. For the case of CIW, we find a different behavior: at $300 \mathrm{~K}, \mathrm{MR}-\mathrm{CIW}$ is positive (dashed blue line), while at $100 \mathrm{~K}$, MR-CIW is negative (open blue circles). In order to explain the sign of MR, some of the authors proposed a simple model [22] based on considering only the AMR contribution of the different kinds 


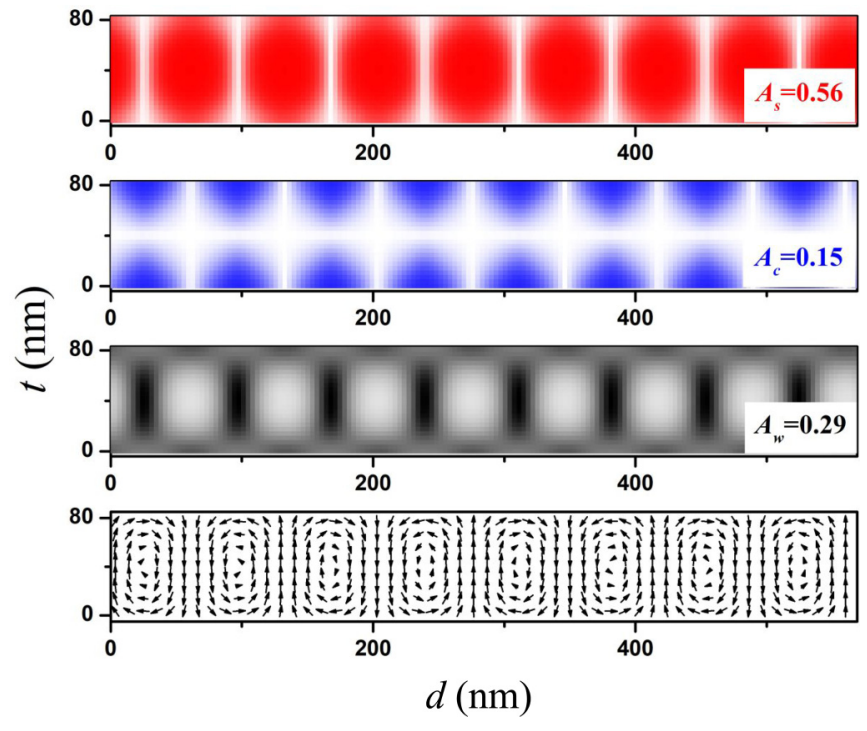

FIG. 5. Cross-sectional view of the stripe domains. Three top panels: color maps for the three components of the magnetization and domain volume ratios calculated by OOMMF package. Bottom panel: magnetization vector along the sample.

of domains as described in Fig. 1(a). Following this model we had success in explaining that MR-CPW can be negative when the electronic current flows perpendicular to the stripes. To understand this behavior, we took into account that the measured resistivity for the three kinds of domains present the following characteristics [22]: $\rho_{s}>\rho_{c}>\rho_{w}$, where subscripts $s, c$, and $w$ correspond to those shown in Fig. 1(a). For the perpendicular geometry, at saturation, the resistivity is that of the $w$-type domains $\left(\rho_{\perp}^{\text {sat }}=\rho_{w}\right)$ which have the lowest resistivity in comparison with the other domains. It means that the system goes from a higher resistivity state composed of the sum of all the domains (when the stripes are set) to another one with lower resistivity because at saturation the only domain present is $A_{w}$, which has the lowest resistivity.

On the other hand, when the current flows parallel to the stripes, the presence of the closure domains gives the possibility for the low-field MR to be positive or negative. To deal with this feature, we proposed a model of parallel resistors [22] taking into account only the AMR contribution. We have found that if the ratio of the volume fraction of the out-of-plane domains to the volume fraction corresponding to closure domains $A_{s} / A_{c}$ is greater than $\sim 0.6$, AMR will be negative, while if such a ratio is lower than $\sim 0.6$, AMR will be positive. We are unable to determine experimentally the domain volume fraction, hence, with the aim of estimating it, we used the micromagnetic calculations performed for our samples. To estimate the volume fraction we have considered that the relative volume of each domain is given by $A_{i}=$ $M_{i}^{2} / M^{2}$, where $i$ labels domain direction, and $A_{s}+A_{c}+A_{w}=$ 1. In Fig. 5, we display the calculated volume fraction for the three kinds of domains at remanence. By taking into account the simulation, the volume ratio of the out-of-plane domain to the closure one $A_{s} / A_{c}$ is $\sim 3.7$ which is very far from the volume ratio required for obtaining the AMR inversion (less than $\sim 0.60$ ). Also, we have performed supplementary calculations by slightly changing the magnetic parameters $\left(M_{s}\right.$ and $K_{\text {PMA }}$ less than $10 \%$ ), in order to study the changes in the volume ratios. We have observed that the volume fraction is almost unaffected when such parameters are modified. This would mean that our simple model of resistors is insufficient for explaining the MR-CIW. In order to obtain a more realistic picture that allows a better quantitative description of the magnetotransport, from the magnetic structure calculated via OOMMF, we proceed to calculate, via the finite element code COMSOL MULTIPHYSICS [41], the resistivity in the perpendicular and parallel configurations for several values of applied field with the aim of calculating the MR ratio. The modeled sample was the same as the one used in micromagnetic simulation, i.e., a $3 \mathrm{D}$ block of $570 \times 570 \times 84 \mathrm{~nm}^{3}$. The mesh was a uniform tetrahedral elements grid, extruded along the stripe axis with a 4:1 aspect ratio. Approximately 112000 elements and 157000 degrees of freedom were used in each simulation. Volume simulation obeyed the $\mathbf{J}=\sigma \mathbf{E}$ law using an isotropic conductivity that was evaluated by interpolating the conductivity map. Boundary conditions were a fixed electric potential difference applied between two opposite edge faces ( $\mathbf{J}=\sigma \mathbf{E}$, Dirichlet condition), and all other boundaries were set to electrical insulation ( $\sigma n=0$, Neumann condition). The current density was integrated over one edge face to achieve the total current flow and the electrical resistance. The results are shown in Fig. 4(b). From this figure, we can observe a good agreement with the experimental data in the perpendicular direction. While, when the electric current flows parallel to the stripes, the MR-CIW sign obtained from the calculations is opposite to the one observed in the experiments at room temperature, showing that the model fails to predict the MRCIW. However, for the case of MR-CIW at low temperature (blue dots), the model predicts the correct MR sign (open circles).

As it was initially stated, the calculation accounts for the AMR behavior within the stripe phase. Then, from the numerical results, it is possible to study which is the MR fraction that corresponds to the AMR. For the case of CPW at room temperature we observe a good agreement with the calculated AMR values. This indicates that the MR observed in this geometry arises mainly from AMR, and other sources can be considered as negligible. On the other hand, in the case of CIW, the calculated MR is positive, showing the opposite behavior with respect to the room-temperature results and indicating the presence of other sources of MR. Another known important contribution to MR in striped thin films is LMR. However, in Ref. [42], the authors show that LMR is negligible when the electronic mean-free path, $l_{\mathrm{mfp}}$, is smaller than the domain size and domain wall. This is our situation because the estimated mean-free path for our system at room temperature is $\sim 8 \mathrm{~nm}$, the domain size about $50 \mathrm{~nm}$, and the domain wall width $\sim 20 \mathrm{~nm}$ [43] (see Fig. 5). Also in that work, it is investigated how the internal field of the stripe domains affects the carrier transport. They found that the net result on the carrier is smoothed due to the large direction dispersion of the internal field generated by the domains and walls; this effect also reduced significantly the LMR contribution.

Taking into account that AMR and LMR cannot explain the sign inversion of MR in CIW geometry, we now discuss 
the presence of DWMR. In order to study this contribution to the MR, we have first to consider extrinsic effects that can mask the intrinsic DWMR contribution. AMR and LMR are discarded as extrinsic effects that can lead to the MR sign inversion. On the one hand, the LMR is negligible in our system as explained before. On the other hand, the contribution coming from the DW's to the AMR was already considered in the micromagnetic calculations and this always presents a negative contribution to MR [see Fig. 4(b)]. Moreover, other extrinsic effects were reported in the literature related with the motion of the carriers close to the DW's in striped systems. One of these is related with the net electric field sense by the electrons that leads to them to follow zigzag trajectories [44]. However, this behavior is only expected in the CPW geometry, being discarded in CIP, which is the geometry where the sign inversion was observed. Also, effects related with coherent scattering [12] and size effects $[45,46]$ have been proposed as DMWR extrinsic sources, that become non-negligible for lengths smaller than the electron mean-free path. However, as mentioned before, the meanfree path in our case is $\sim 8 \mathrm{~nm}$ and the domain size is $\sim 50 \mathrm{~nm}$, indicating that those contributions can be discarded. Regarding the intrinsic DWMR, it arises in the literature as an important source of MR [11,16,18,19]. In order to evaluate if the intrinsic DWMR contributes to the observed MR in our system, it must fulfill two conditions. On one hand, this must be negative at room temperature and, on the other hand, this contribution must change its sign with temperature. van Gorkom et al. [20] have developed a model where the DWMR sign depends on the difference of the both spin channels relaxation time, i.e., DWMR ratio $\propto\left(\tau^{\uparrow}-\tau^{\downarrow}\right)$. The resistivity of our system varies from $47 \mu \Omega \mathrm{cm}$ at $100 \mathrm{~K}$ to $55 \mu \Omega \mathrm{cm}$ at $300 \mathrm{~K}$ [47], where the thermal-activated magnon and phonon scattering lead to the resistivity increase. The scattering is spin dependent, therefore, the temperature dependence of $\tau^{\uparrow}$ and $\tau^{\downarrow}$ is not the same, giving the possibility to $\left(\tau^{\uparrow}-\tau^{\downarrow}\right)$ to change in sign. Although this model proposed by van Gorkom et al. would explain the behavior observed in our experiments, to know the $\tau^{\uparrow(\downarrow)}$ values is needed for a proper addressing. To determine the spin-dependent scatter- ing rates is not trivial and a quantitative description of the polarized band structure is required. However, a theoretical study of the transport properties focused on band-structure calculations is still lacking in $\mathrm{Fe}_{1-x} \mathrm{Ga}_{x}$ thin films.

\section{CONCLUSIONS}

In this work, we have demonstrated experimentally by means of XRMS the existence of closure domains in $\mathrm{Fe}_{0.8} \mathrm{Ga}_{0.2}$ thin films where stripes are present. From these experimental data, with the aid of micromagnetic simulations, we have determined that closure domains occupy $(55 \pm 15) \%$ of the surface magnetization. The micromagnetic calculations indicate that the model of volume fraction proposed in Ref. [22] is suitable when the electronic current flows perpendicular to the stripes, suggesting that the main contribution to MR-CPW is from AMR. For current flowing parallel to the stripes, MR-CIW, such a model fails to explain the sign of MR at room temperature, while at low temperature, the model predicts the experimental observed behavior. An intrinsic contribution to the MR from domain walls appears as the most plausible explanation in order to give account the positive MR observed. Further detailed magnetotransport experiments as a function of temperature, band-structure calculation, and also modeling of scattering rates are needed in order to go into depth in terms to understanding MR in striped $\mathrm{Fe}_{1-x} \mathrm{Ga}_{x}$ thin films. Finally, we remark that we have put in evidence the importance of using x-ray resonant magnetic scattering for the determination of thin-film properties related with the magnetic structure.

\section{ACKNOWLEDGMENTS}

The authors gratefully acknowledge S. Bustingorry for fruitful discussions about this work and also R. Benavides, C. Pérez, and M. Guillén for technical support. B.P., M.B., and J.M. acknowledge partial financial support by PIP Grant No. 11220120100250CO, PICT Grant No. 2013-0401, and SIIP Grant No. 06/C510.
[1] C. Liu, S. Wu, J. Zhang, J. Chen, J. Ding, J. Ma, Y. Zhang, Y. Sun, S. Tu, H. Wang, et al., Nat. Nanotechnol. 14, 691 (2019).

[2] M. Barturen, B. Rache Salles, P. Schio, J. Milano, A. Butera, S. Bustingorry, C. Ramos, A. J. A. de Oliveira, M. Eddrief, E. Lacaze, F. Gendron, V. H. Etgens, and M. Marangolo, Appl. Phys. Lett. 101, 092404 (2012).

[3] M. Barturen, J. Milano, M. Vásquez-Mansilla, C. Helman, M. A. Barral, A. M. Llois, M. Eddrief, and M. Marangolo, Phys. Rev. B 92, 054418 (2015).

[4] N. Saito, H. Fujiwara, and Y. Sugita, J. Phys. Soc. Jpn. 19, 1116 (1964).

[5] A. Hubert and R. Schäfer, Magnetic Domains: The Analysis of Magnetic Microstructures (Springer, Berlin, 1998).

[6] Y. Murayama, J. Phys. Soc. Jpn. 21, 2253 (1966).

[7] S. Tacchi, S. Fin, G. Carlotti, G. Gubbiotti, M. Madami, M. Barturen, M. Marangolo, M. Eddrief, D. Bisero, A. Rettori, and M. G. Pini, Phys. Rev. B 89, 024411 (2014).
[8] C. Donnelly, M. Guizar-Sicairos, V. Scagnoli, S. Gliga, M. Holler, J. Raabe, and L. J. Heyderman, Nature (London) 547, 328 (2017).

[9] J. B. Kortright, J. Electron Spectrosc. 189, 178 (2013).

[10] J. F. Gregg, W. Allen, K. Ounadjela, M. Viret, M. Hehn, S. M. Thompson, and J. M. D. Coey, Phys. Rev. Lett. 77, 1580 (1996).

[11] C. H. Marrows, Adv. Phys. 54, 585 (2005).

[12] A. D. Kent, J. Yu, U. Rüdiger, and S. S. Parkin, J. Phys.: Condens. Matter 13, R461 (2001).

[13] U. Ruediger, J. Yu, S. Zhang, A. D. Kent, and S. S. P. Parkin, Phys. Rev. Lett. 80, 5639 (1998).

[14] U. Rüdiger, J. Yu, L. Thomas, S. S. P. Parkin, and A. D. Kent, Phys. Rev. B 59, 11914 (1999).

[15] L. Klein, Y. Kats, A. F. Marshall, J. W. Reiner, T. H. Geballe, M. R. Beasley, and A. Kapitulnik, Phys. Rev. Lett. 84, 6090 (2000). 
[16] R. M. Reeve, A. Loescher, H. Kazemi, B. Dupé, M.-A. Mawass, T. Winkler, D. Schönke, J. Miao, K. Litzius, N. Sedlmayr, I. Schneider, J. Sinova, S. Eggert, and M. Kläui, Phys. Rev. B 99, 214437 (2019).

[17] M. Viret, D. Vignoles, D. Cole, J. M. D. Coey, W. Allen, D. S. Daniel, and J. F. Gregg, Phys. Rev. B 53, 8464 (1996).

[18] P. M. Levy and S. Zhang, Phys. Rev. Lett. 79, 5110 (1997).

[19] G. Tatara and H. Fukuyama, Phys. Rev. Lett. 78, 3773 (1997).

[20] R. P. van Gorkom, A. Brataas, and G. E. W. Bauer, Phys. Rev. Lett. 83, 4401 (1999).

[21] V. A. Gopar, D. Weinmann, R. A. Jalabert, and R. L. Stamps, Phys. Rev. B 69, 014426 (2004).

[22] M. Granada, S. Bustingorry, D. E. Pontello, M. Barturen, M. Eddrief, M. Marangolo, and J. Milano, Phys. Rev. B 94, 184435 (2016).

[23] M. Eddrief, M. Marangolo, V. H. Etgens, S. Ustaze, F. Sirotti, M. Mulazzi, G. Panaccione, D. H. Mosca, B. Lépine, and P. Schieffer, Phys. Rev. B 73, 115315 (2006).

[24] M. Eddrief, M. Marangolo, S. Corlevi, G.-M. Guichar, V. H. Etgens, R. Mattana, D. H. Mosca, and F. Sirotti, Appl. Phys. Lett. 81, 4553 (2002).

[25] M. Eddrief, Y. Zheng, S. Hidki, B. Rache Salles, J. Milano, V. H. Etgens, and M. Marangolo, Phys. Rev. B 84, 161410 (2011).

[26] M. Sacchi, C. Spezzani, P. Torelli, A. Avila, R. Delaunay, and C. F. Hague, Rev. Sci. Instrum. 74, 2791 (2003).

[27] J. Torrejon, G. Malinowski, M. Pelloux, R. Weil, A. Thiaville, J. Curiale, D. Lacour, F. Montaigne, and M. Hehn, Phys. Rev. Lett. 109, 106601 (2012).

[28] M. J. Donahue and D. G. Porter, oомmF User's Guide, Version 1.0, Interagency Report NISTIR 6376 (National Institute of Standards and Technology, Gaithersburg, MD, 1999).

[29] I. S. Camara, S. Tacchi, L.-C. Garnier, M. Eddrief, F. Fortuna, G. Carlotti, and M. Marangolo, J. Phys.: Condens. Matter 29, 465803 (2017).

[30] The specular spot is enlarged by the presence of weak nonmonochromatic low-energy photons (the fundamental energy of the wiggler source is about $5 \mathrm{eV}$ ) that are reflected with high efficiency from the sample.

[31] H. Dürr, E. Dudzik, S. Dhesi, J. Goedkoop, G. Van der Laan, M. Belakhovsky, C. Mocuta, A. Marty, and Y. Samson, Science 284, 2166 (1999).

[32] S. Flewett et al. (unpublished).

[33] S. Flewett, T. J. A. Mori, A. Ovalle, S. Oyarzún, A. Ibáñez, S. Michea, J. Escrig, and J. Denardin, Phys. Rep. 9, 14823 (2019).

[34] S. Flewett, D. Mishra, T. J. A. Mori, C. M. Günther, J. C. Denardin, S. Oyarzún, S. Michea, D. Engel, M. Fohler, T. C. R. Rocha, A. Ovalle F., L. T. Núñez A., B. Pfau, J. Escrig, and S. Eisebitt, Phys. Rev. B 95, 094430 (2017).

[35] N.-T. D. Loh, S. Eisebitt, S. Flewett, and V. Elser, Phys. Rev. E 82, 061128 (2010).

[36] Z. Q. Qiu and S. D. Bader, Rev. Sci. Instrum. 71, 1243 (2000).

[37] C. Spezzani, F. Fortuna, R. Delaunay, H. Popescu, and M. Sacchi, Phys. Rev. B 88, 224420 (2013).

[38] The x-ray beam is skimmed by a $500-\mu \mathrm{m}$ pinhole placed at $450 \mathrm{~mm}$ from the sample, giving a transverse coherence of $\sim 1$ $\mu \mathrm{m}$.

[39] M. Sacchi, C. Spezzani, A. Carpentiero, M. Prasciolu, R. Delaunay, J. Lüning, and F. Polack, Rev. Sci. Instrum. 78, 043702 (2007).

[40] The asymmetry ratio maximum occurs at only 20-nm domain wall width due to the transverse MOKE component being greater in magnitude to the longitudinal and polar MOKE components at this particular incidence angle.

[41] COMSOL MULTIPHYSICS Reference Manual, version 4.4, COMSOL, Inc.

[42] I. Knittel and U. Hartmann, J. Magn. Magn. Mater. 294, 16 (2005).

[43] M. D. P. Martínez, J. Milano, M. Eddrief, M. Marangolo, and S. Bustingorry, J. Phys.: Condens. Matter 28, 136001 (2016).

[44] L. Berger, Phys. Rev. B 2, 4559 (1970).

[45] E. Sondheimer, Adv. Phys. 1, 1 (1952).

[46] J. Milano and A. M. Llois, J. Appl. Phys. 102, 013705 (2007).

[47] D. Pontello, Technical Report, Universidad Nacional de Cuyo, Instituto Balseiro, 2016 (unpublished). 